\title{
Efficacy of transabdominal ultrasound examination in the diagnosis of early pregnancy complications in an emergency department
}

\author{
T W Wong, C C Lau, A Yeung, L Lo, C M Tai
}

\begin{abstract}
Objective-To assess the value of ultrasound in an emergency department in the diagnosis of early pregnancy complications, the efficacy of a study protocol in identifying ectopic pregnancies, and the agreement on ultrasound findings among emergency department staff and gynaecologists.
\end{abstract}

Methods-All women presenting with early pregnancy complications had a transabdominal ultrasound scan performed by the attending doctor and checked by a senior doctor. The ultrasound findings were classified as normal intrauterine pregnancy (IUP), probable abnormal pregnancy, definite ectopic pregnancy, no definite IUP, and other. Women with clinical and ultrasound findings compatible with threatened abortion were referred to a gynaecologist, or were admitted if findings suggested abnormal or ectopic pregnancy, or if a definite IUP could not be confirmed on ultrasound scan. For patients who were admitted or referred, a transvaginal ultrasound scan was performed by the attending gynaecologist. The findings of the gynaecologist were used as the gold standard.

Results-151 cases were enrolled during a four month study period. Ultrasound findings in the emergency department included definite IUP in $95(63 \%)$, probable abnormal IUP in 20 (13\%), no definite IUP in $23(21 \%)$, and other findings in four (3\%). For evaluating the presence or absence of IUP, sensitivity of the initial scan was $82 \%$ (95\% confidence interval $76 \%$ to $88 \%)$ and specificity $92 \%(88 \%$ to $96 \%$ ). Agreement between junior and senior emergency department doctors on their ultrasound findings was $81 \%$ (75\% to $87 \%$ ) and between emergency department senior staff and gynecologists $85 \%$ (79\% to $91 \%)$. The diagnoses made in the emergency department were thought to be compatible with the final assessments by gynaecologist in $72 \%(65 \%$ to $79 \%)$. Using either no definite IUP or other findings as a positive screening test for ectopic pregnancy, the sensitivity, specificity, positive predictive value, and negative predictive value were $80 \%$ ( $74 \%$ to $86 \%), 78 \%$ (71\% to $85 \%), 12 \%(7 \%$ to $17 \%)$, and $99 \%(97 \%$ to $100 \%)$, respectively.

Conclusions-Transabdominal ultrasound performed in the emergency department is useful in screening for early pregnancy complications. Ectopic pregnancy should be suspected when no IUP is found on preliminary scanning.

( Accid Emerg Med 1998;15:155-158)

Keywords: ectopic pregnancy; pregnancy complications, ultrasound

Ectopic pregnancy is the most important gynaecological emergency that should not be missed in the emergency department. Various clinical and laboratory features are used to diagnose ectopic pregnancy but none is foolproof. On the basis of clinical features alone, as many as $45 \%$ of ectopic pregnancies may not be diagnosed at initial presentation. ${ }^{1}$ Ultrasound has increasingly been used by doctors working in emergency departments in the diagnosis of various acute abdominal conditions. ${ }^{2}$ The use of ultrasound at the bedside for targeted examination has become popular. A prospective study of ultrasonography in the emergency department by emergency physicians in the USA showed that the three most commonly performed ultrasound studies were for gall bladder disease (53\%), pregnancy related conditions $(28 \%)$, and abdominal aortic aneurysms (7\%). The overall diagnostic accuracy of emergency physicians was found to be high. ${ }^{3}$ Ultrasound is costeffective in narrowing the differential diagnosis and excluding some potentially serious conditions. ${ }^{4}$ Pregnancy complications in the first trimester are common presentations in the emergency department and ultrasonography can be very useful in making the diagnosis. In particular, it may be an important tool to identify ectopic pregnancy, a diagnosis we cannot afford to miss.

The aim of our study was to assess the value of ultrasound in the diagnosis of pregnancy complications in the hands of emergency department doctors, the efficacy of the study protocol in identifying ectopic pregnancies, and the agreement on ultrasound findings among trainees, senior staff members of the emergency department, and gynaecologists.

Methods

All women presenting to our department during the study period from 15 February to 15 June 1996 with early pregnancy (less than 16 week gestation by date) were included. Inclusion criteria were a positive urine pregnancy test and a complaint of vaginal bleeding or lower abdominal pain, or both. Patients who were haemodynamically unstable were excluded. Patients who were referred by a private 
Table 1 Definitions of ultrasound findings

Definite intrauterine pregnancy

- Gestation sac with sonolucent centre $>5 \mathrm{~mm}$

- Thick concentric echogenic ring within endometrium

- Fetal pole \pm yolk sac

Probable abnormal intrauterine pregnancy

- GS > $16 \mathrm{~mm}$ without fetal pole

- $\mathrm{CRL}>5 \mathrm{~mm}$ without cardiac pulsation

Definite ectopic pregnancy

- Thick echogenic ring-like structure with fetal pole outside of uterus

No definite intrauterine pregnancy

- None of the criteria above

Other findings

- Fluid in pouch of Douglas

- Blood clots inside endometrial cavity

- Subchorionic bleeding

CRL, crown-rump length; GS, gestation sac.

practitioner and had already had an ultrasound examination done were also excluded.

The date of the last menstrual period was ascertained and the gestational age calculated. A transabdominal ultrasound scan was performed by the attending doctor using an Aloka SD-500 ultrasound system equipped with a 3.5 $\mathrm{MHz}$ transducer. A full bladder was required. On the job training was provided for all junior staff.

Patients' demographic data, clinical presentation, and ultrasound findings were recorded on a study form. The ultrasound findings were categorised as normal intrauterine pregnancy (IUP), probable abnormal pregnancy, definite ectopic pregnancy, no definite IUP, and other. Ultrasound finding categories were as defined in table 1. Ultrasound scan findings were entered by the trainee on the study form, and a member of senior staff scanned the woman again to countercheck the findings. Agreement between junior and senior staff could be determined from the study form. Duplicates of the ultrasound findings were printed and appended to the patient's record and to the study form.

Women with clinical and ultrasound findings compatible with threatened abortion were referred to the gynaecology outpatient clinic. They were admitted if findings suggested abnormal or ectopic pregnancy, or if a definite IUP could not be confirmed on the ultrasound scan. In women admitted to the gynaecological ward, transvaginal ultrasound for suspected ectopic pregnancy was usually performed immediately by a gynaecologist and within 24 hours for other pregnancy complications. Patients referred to the outpatient department were usually seen within a week and an ultrasound examination performed if deemed necessary by the attending gynaecologist. The findings of the gynaecologist were used as the gold standard. Agreement between senior

Table 2 Ultrasound (US) diagnosis $v$ final diagnosis

\begin{tabular}{lllll}
\hline & \multicolumn{2}{l}{ Final diagnosis } & & \\
\cline { 2 - 5 } US diagnosis & $\begin{array}{l}\text { Threatened } \\
\text { abortion }\end{array}$ & Missed abortion & $\begin{array}{l}\text { Spontaneous } \\
\text { abortion }\end{array}$ & Ectopic \\
\hline IUP & 56 & 25 & 7 & 1 \\
Abnormal IUP & 3 & 17 & - & - \\
No Definite IUP & 7 & 16 & 7 & 4 \\
\hline
\end{tabular}

IUP, intrauterine pregnancy. emergency department doctors and gynaecologists could then be determined from the gynaecologists' ultrasound reports. Compatibility of the admitting emergency department diagnoses with gynaecology unit discharge diagnoses was assessed based on the patients' medical records. All data were analysed using the SPSSPC package on an IBM compatible computer.

\section{Results}

During the four month study period, 151 cases were enrolled. The average age of the women was 30 years and the mean gestation age of the fetuses was nine weeks. The presenting complaint was vaginal bleeding alone in 98 cases $(65 \%)$ and bleeding with concomitant abdominal pain in $48(32 \%)$. A principal complaint of abdominal pain alone was elicited in only five patients $(3.3 \%)$.

The ultrasound findings of the emergency department senior staff included definite IUP in 95 cases $(63 \%)$, probable abnormal IUP in $20(13 \%)$, no definite IUP in $32(21 \%)$, and other findings in four $(3 \%)$. In the evaluation of the presence or absence of IUP, the initial scan had a sensitivity of $82 \%$ (95\% confidence interval $76 \%$ to $88 \%$ ) and a specificity of $92 \%$ (88\% to $96 \%)$.

The agreement between emergency department junior and senior doctors on their ultrasound findings was $81 \%$ ( $75 \%$ to $87 \%)$. In the 136 cases where transvaginal ultrasound was performed by the gynaecologist, the findings of emergency department senior staff were thought to be compatible with their findings in $85 \%$ ( $79 \%$ to $91 \%)$. The compatibility in ultrasound findings was higher $(88 \%(81 \%$ to $95 \%)$ ) when the gestation age was beyond seven weeks. For women who were admitted, compatibility was only $81 \%$ ( $73 \%$ to $89 \%)$, lower than in the referred group ( $94 \%$ ( $86 \%$ to $100 \%)$.

Missed abortion was the admitting diagnosis in 57 cases $(38 \%)$ and threatened abortion in $34(23 \%)$. Ectopic pregnancy was diagnosed in three women $(2 \%)$ on the basis of clinical and ultrasound features, and $10(7 \%)$ were admitted to rule out ectopic pregnancy. Incomplete abortion was the cause for admission in only four patients (3\%). Forty three women $(29 \%)$ with a diagnosis of threatened abortion were referred for outpatient follow up but eight defaulted.

The diagnoses made in the emergency department were thought to be compatible with the final assessments by gynaecologists in $72 \%$ (65\% to $79 \%)$. The compatibility rate was higher $(75 \%(67 \%$ to $83 \%)$ if the gestational age was more than seven weeks. The agreement in diagnosis was highest $(92 \%$ (83\% to $99 \%$ ) for the referred group and lowest $(65 \%$ ( $56 \%$ to $74 \%$ ) in the admitted group. The relation between the admitting ultrasound classification and discharge diagnosis is given in table 2.

Five women $(3.3 \%)$ had a final diagnosis of ectopic pregnancy. Of these five, three were admitted with a diagnosis of ectopic pregnancy, and one was admitted to rule out 
ectopic pregnancy. The fifth was referred for outpatient follow up with a provisional diagnosis of threatened abortion.

Using the ultrasound findings of no definite IUP or other findings as a positive screening test for ectopic pregnancy, the sensitivity, specificity, positive predictive value, and negative predictive value were $80 \%$ ( $74 \%$ to $86 \%$ ), $78 \%(71 \%$ to $85 \%), 12 \%$ ( $7 \%$ to $17 \%)$, and $99 \%$ ( $97 \%$ to $100 \%)$, respectively.

The patient who was not diagnosed initially as having an ectopic pregnancy presented to the emergency department with a history of vaginal bleeding and mild abdominal pain at five weeks of gestation. A sac which was thought to be a gestation sac was noted on transabdominal scan in the emergency department. The patient was referred to the gynaecological outpatient clinic and was seen the next day. She was subsequently admitted and had a transvaginal ultrasound the following day which showed a small cystic space of around 3 $\mathrm{mm}$ in diameter just adjacent to the endometrial cavity. Serum $\beta$ human chorionic gonadotrophin ( $\beta$-hCG) was ordered and another scan was done on the next day, which revealed no definite intrauterine sac. Abdominal pain had subsided after admission and the patient was observed and serial serum $\beta$-hCG levels done. One week after admission an ultrasound scan was repeated and a right tubal mass with a small amount of fluid was noted. A diagnostic laparoscopy was performed and an unruptured tubal pregnancy was found at the ampulla region of the right fallopian tube. A right salpingectomy was performed and the patient had an uneventful recovery.

\section{Discussion}

In the USA, the reported rate of ectopic pregnancy is 16 per $1000 .^{5}$ In the emergency department population, the rate of ectopic pregnancy reported varies from $7 \%$ to $13 \% .^{6-9}$ In our study, the incidence of ectopic pregnancy was only $3.3 \%$. We were not alerted by our gynaecologists of any missed cases not included in the study. Even if all the eight patients who had defaulted follow up had ectopic pregnancy, the incidence would only be about $8.6 \%$. With our lower incidence of ectopic pregnancy, making a correct diagnosis becomes all the more difficult. We will need a very sensitive tool to identify an ectopic pregnancy. For women with a positive pregnancy test and no IUP, a sensitivity of $21-84 \%$ and a specificity of $93-99.5 \%$ have been reported with the use of transvaginal ultrasound for screening for ectopic pregnancy. ${ }^{5}$ Our sensitivity of $80 \%$ and specificity of $78 \%$ were not as good as in some other studies performed in the emergency department. ${ }^{8-10}$ However, this could be a result of differences in the protocol. For example, in a study by Mateer et $a l^{8}$ only $12(75 \%)$ of the 16 patients with ectopic pregnancy were diagnosed with the initial scan. Thus the sensitivity of their ultrasound scan at initial contact was only $75 \%$. The four patients not diagnosed initially had $\beta$-hCG levels of less than $2000 \mathrm{mIU} / \mathrm{ml}$ and the diagnosis of ectopic pregnancy was confirmed by serial ultrasound and $\beta$-hCG measurements.

Our lower specificity could be owing to our use of transabdominal scanning, which is less reliable at early gestations. Some patients classified as having no definite IUP by transabdominal scan might have an IUP confirmed if transvaginal ultrasound were available. Transabdominal ultrasound was also affected more by other factors such as obesity. In a study of the use of ultrasound screening for ectopic pregnancy by radiologists, transvaginal ultrasound was required after a preliminary transabdominal scan in $55 \%$ of the cases to reach a diagnosis. Transvaginal ultrasound was used to confirm the viability of an IUP, to further evaluate those cases lacking an IUP, and to characterise an adnexal mass. ${ }^{\circ}$

Serial $\beta$-hCG is often used to improve the specificity of ultrasound screening for ectopic pregnancy. For example, if the discriminatory level of $\beta$-hCG is set at $4500 \mathrm{mIU} / \mathrm{ml}$, the specificity of absent IUP will be $100 \%$ but the sensitivity will be low. ${ }^{10}$ Using an hCG value of $1500 \mathrm{mIU} / \mathrm{ml}$ as the discriminatory level for transvaginal ultrasound examination, the diagnosis of ectopic pregnancies on the day of presentation to the emergency department could be made by a radiologist with a sensitivity of $81.6 \%$ and a specificity of $100 \% .^{6}$ In that study, women with a $\beta$-hCG less than 1500 $\mathrm{mIU} / \mathrm{ml}$ were followed up with serial $\beta$-hCG measurement and only those reaching the discriminatory level were scanned. The addition of progesterone has been advocated by some. However, progestorone concentration, though usually raised in a viable pregnancy, has not been found to be a useful clinical predictor of ectopic pregnancy. ${ }^{11}$ In an emergency department like ours, where progesterone and $\beta$-hCG are not available as an urgent test, the use of ultrasound seems to be comparable, in terms of sensitivity, with protocols using additional blood tests as screening tools. Specificity is lower and there will be more admissions. However, in a recent study by Kaplan et al, patients with a $\beta$-hCG level of $1000 \mathrm{mIU} / \mathrm{ml}$ or less had a fourfold increase in the risk of ectopic pregnancy. In the group of patient with indeterminate ultrasound findings and a $\beta$-hCG value of $1000 \mathrm{mIU} / \mathrm{ml}$ or less, the sensitivity and specificity for ultrasound were $67 \%$ and $74 \%$ respectively. ${ }^{7}$ Thus the practice of sending patients with a $\beta$-hCG value below the discriminatory level home for serial testing without ultrasound is called into question.

Ultrasound is an operator dependent diagnostic tool and is not infallible, even in good hands. The agreement between senior and junior staff was only $81 \%$ in our study. Junior doctors should not be relied upon to perform the study independently. The woman whose ectopic pregnancy was not suspected initially and who was referred for follow up by the gynaecologists probably had a pseudosac. Pseudosacs, which are a result of a prominent decidual reaction surrounding centrally located endometrial fluid, have been reported in $5-10 \%$ of patients with ectopic pregnancy. ${ }^{5}$ 
Using videotape as the recording medium, Mateer et al have found that the findings of emergency physicians were in agreement with consultant gynaecologist in $93 \%$ of the scans. ${ }^{8}$ In our study, the agreement between senior emergency department doctors and gynaecologists was only $85 \%$. However, some of the discrepancies might be caused by a change in the status of the fetus, since the scan was not repeated immediately. The fact that gynaecologists were using transvaginal ultrasound, which is more sensitive in early pregnancy, might also account for some of the discrepancy. Agreement was slightly better ( $88 \%$ ) if gestation was beyond seven weeks. In the study by Mateer et $a l^{8}$ the initial endovaginal scans by the emergency physician for the evaluation of the presence or absence of IUP had a sensitivity of $99 \%(97 \%$ to $100 \%)$ and a specificity of $93 \%$ $(80 \%$ to $100 \%){ }^{8}$ Again, our sensitivity $(82 \%)$ and specificity $(92 \%)$ were lower, possibly owing to the limitations of using transabdominal scan. The additional capability of performing transvaginal ultrasound in the emergency department would be a worthwhile investment to improve our diagnostic accuracy. The use of transvaginal ultrasound as the initial examination has been found to be more cost-effective, since only $9 \%$ of patients would require additional transabdominal scanning, whereas $74 \%$ would require transvaginal ultrasound if transabdominal scan was the initial examination. $^{12}$

Agreement on diagnosis (92\%) was highest in women with an ultrasound diagnosis of IUP who were referred for outpatient follow up with a diagnosis of threatened abortion. This is comparable with the $90 \%$ agreement reported by Mateer et al. ${ }^{8}$ Agreement was low (65\%) in the admitted group and this was partly owing to the difficulty in confirming a missed abortion at early gestation using transabdominal ultrasound. However, screening for ectopic and abnormal pregnancy rather than reaching a definitive diagnosis is probably more important in the initial emergency department encounter.

CONCLUSIONS

Transabdominal ultrasound in the emergency department is useful in screening for early pregnancy complications. Ectopic pregnancy should be suspected when no IUP is found on preliminary scanning.

We thank Ms Patty Lee for her help in the retrieval of patient records and data entry.

1 Stovall TG, Kellerman AL, Ling FW, Buster JE. Emergency department diagnosis of ectopic pregnancy. Ann Emerg Med 1990;19:1098-103.

2 Heller MB, Verdile VP. Ultrasonography in emergency medicine. Emerg Med Clin North Am 1992;10:27-45.

3 Schlager D, Lazzareschi G, Whitten D, Sanders AB. A prospective study of ultrasonography in the ED by emergency physicians. Am J Emerg Med 1994;12:185-9.

4 Shea DJ, Aghababian RV. The efficacy of abdominal and pelvic ultrasound in the emergency department. Ann Emerg Med 1984;13:311-16.

5 Frates MC, Laing FC. Sonographic evaluation of ectopic pregnancy: an update. Am J Radiol 1995;165:251-9.

6 Barnhart $\mathrm{K}$ Mennuti MT, Benjamin I, Jacobson S Goodman D, Coutifaris C. Prompt diagnosis of ectopic pregnancy in an emergency department setting. Obstet pregnancy in an emergen
Gynecol 1994;84:1010-15.

7 Kaplan BC, Dart RG, Moskos M, Kuligowska E, Chun B, Adel-Hamid M, et al. Ectopic pregnancy: prospective study with improved diagnostic accuracy. Ann Emerg Med 1996; 28: $10-17$.

8 Mateer JR, Aiman EJ, Brown MH, Olson DW. Ultrasonographic examination by emergency physicians of patients at risk for ectopic pregnancy. Acad Emerg Med 1995;2:86773.

9 Braffman BH, Coleman BG, Ramchandani P, Arger PH, Nodine CF, Kinsmore BJ, et al. Emergency department screening for ectopic pregnancy: a prospective US study. Radiology 1994;190:797-802.

10 Ankum WM, Van der Veen F, Hamerlynck JV, Lammes FB. Transvaginal sonography and human chorionic gonadotrophin measurements in suspected ectopic pregnancy: a detailed analysis of a diagnostic approach. Hum Reprod 1993;8:1307-11.

11 Ledger WL, Sweeting VM, Chatterjee S. Rapid diagnosis of early ectopic pregnancy in an emergency gynecological service: are measurements of progesterone, intact and free beta human chorionic gonadotrphin helpful? Hum Reprod 1994;9:157-60.

12 Hazlett KS, Wagner A, Guidi C, Victoria D, Weir S, Wise S. Cost effectiveness of pelvic sonogram in the emergency room: endovaginal vs. transabdominal examination. Emerg Radiol 1996;3:231-5. 